\title{
MAGNETIC BEHAVIOUR OF NON-CONTACTING NI NANOPARTICLES ENCAPSULATED IN VERTICALLY ALIGNED CARBON NANOTUBES
}

\author{
$\underline{\text { L. Elbaile }}^{\underline{a}}{ }_{2}$ J.A. García ${ }^{a}$, E. Bertran $^{b}$, J. García-Céspedes $^{b}$ and A. Svalov ${ }^{c}$
}

a Departamento de Física, Universidad de Oviedo, c/ Calvo Sotelo s/n 33007 Oviedo, Spain b Grupo FEMAN, IN2UB, Universitat de Barcelona, Martí i Franquès 1, E08028 Barcelona Spain.

c Ural State University, Institute of Physics and Applied Mathematics, Lenin Ave. 51620083

Ekaterinburg, Russia

elbaile@uniovi.es

Magnetic properties of carbon nanotubes (MF-CNT) obtained by plasma-enhanced chemical vapour deposition (PECVD) have been studied [1]. The growth of these nanotubes has been activated from Ni catalyst nanoparticles. Samples consist of Ni nanoparticles encapsulated at the tip of vertically aligned multiwalled carbon nanotubes (VACNTs) forming an homogeneous and dense large-area monolayer of magnetically isolated (non-contacting) nanoparticles. Figure 1 shows SEM micrograph of aligned CNTs grown on Si using Ni as catalyst and the diameter and mass distribution (normalised) histograms.

The magnetic characterisation has been performed by a Physical Property Measurement system (PPMS-14 T) in the temperature range of 5-300 K with magnetic fields up to $9 \mathrm{~T}$. Figure 2 shows the hysteresis loops of the Ni particles in carbon nanotubes in the 5-300 K temperature range.

The results show that the wide size range $(30-180 \mathrm{~nm})$ of the particles originates the coexistence of blocked and superparamagnetic particles that leads into an intense ferromagnetic behaviour of the whole assembly [2]. At $5 \mathrm{~K}$ temperature the magnetization saturation is reached at an applied magnetic field of $\mu_{0} H=8 \mathrm{~T}$. The coercivity decreases monotonically with increasing temperature and the value for the intrinsic coercivity is 250 Oe. The encapsulation of Ni nanoparticles by VACNTs preserves them from aggregation, this makes possible a tuning of the coercivity by controlling size distribution of particle monolayers. Torque measurements in the plane of the substrate and in the perpendicular plane, indicate the absence of preferential orientation of the Ni particles.

\section{References:}

[1] H. Li, N. Zhao, Ch. He, Ch. Shi, X. Du and J. Li, J. of Alloys and Compounds 465 (2008) 51.

[2] M.E. McHenry, Phys. Rev. B, 49 (1994) 11358. 
Fig.1

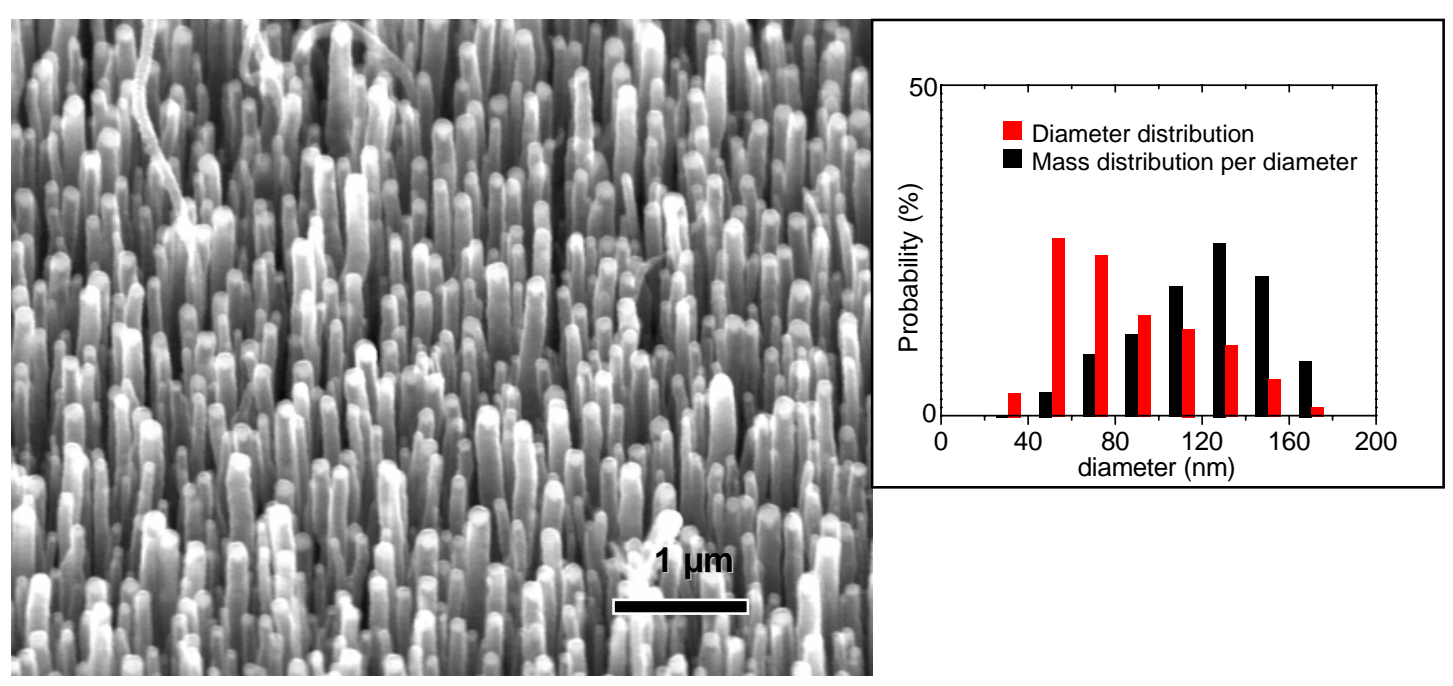

Fig.2

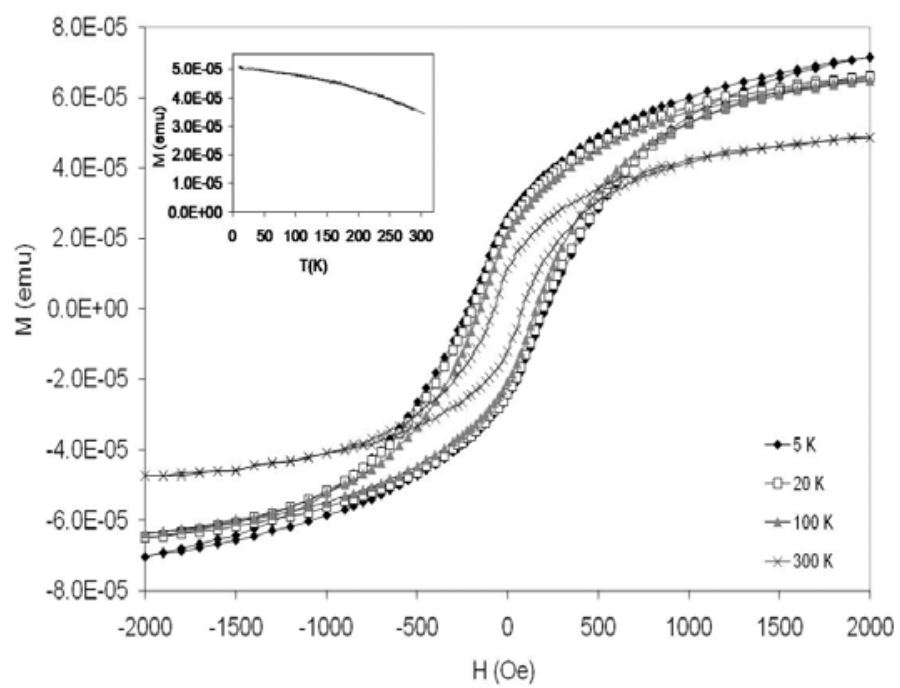

